\section{Topotecan beim platinsensitiven Ovarialkarzinom}

\author{
In einer randomisierten Phase-III-Studie wurden die Effizienz und Sicherheit \\ von Topotecan und Carboplatin bei platinsensitiven rezidivierten Ovarial- \\ karzinomen mit der von platinbasierten Standardkombinationen verglichen.
}

\begin{abstract}
ei Patientinnen mit rezidiviertem plaD tinsensitivem Ovarialkarzinom werden verschiedene platinbasierte Regimes empfohlen. Der Topoisomerase-I-Inhibitor Topotecan ist in der palliativen Therapie gut etabliert und bei platinresistenten Tumoren ähnlich effizient wie Paclitaxel und pegyliertes liposomales Doxorubicin. In der Studie erhielten insgesamt 550 Patientinnen randomisiert Topotecan/Carboplatin (TC) oder ein Standardregime mit Paclitaxel/Carboplatin (PC), Gemcitabin/Carboplatin (GC) oder pegyliertem liposomalem Doxorubicin/Carboplatin (PLDC). Primä-
\end{abstract}

rer Endpunkt war das progressionsfreie Überleben (PFS) nach 12 Monaten, sekundäre Endpunkte unter anderem Gesamtüberleben (OS) und Ansprechrate.

Nach median 20 Monaten betrug die Rate für das 12-Monats-PFS 37,0\% im TCund 40,2\% im Standardarm ( $\mathrm{p}=0,470)$. Auch abhängig von Alter, Zahl der Vortherapien und Histologie (seröse bzw. nichtseröse Tumoren) ergaben sich keine Unterschiede. Insgesamt starben 262 (47,6\%) Patientinnen, die Krankheit war bei $426(77,5 \%)$ progredient. Die generelle Ansprechrate betrug 73,1\% im TCArm und 75,1\% im Standardarm ( $\mathrm{p}=$
0,149). Das mediane PFS lag im TC-Arm bei 10, im Standardarm bei 11 Monaten $(\mathrm{p}=0,414)$. Insgesamt betrug das mediane OS 27 Monate, im TC-Arm 25, im Standardarm 31, auch hier gab es keinen signifikanten Unterschied ( $\mathrm{p}=0,163)$. Hämatologische Toxizitäten vom Grad 3/4 waren im Standardarm signifikant häufiger als im experimentellen Arm ( $\mathrm{p}$ $<0,001)$. Das Fazit: Bei platinsensitiven rezidivierten Ovarialkarzinomen war die Kombination aus Topotecan plus Carboplatin gegenüber Standardregimes zwar besser verträglich, konnte aber weder PFS noch OS verbessern. Kathrin von Kieseritzky

Sehouli J et al. Topotecan plus carboplatin versus standard therapy with paclitaxel plus carboplatin (PC) or gemcitabine plus carboplatin (GC) or pegylated liposomal doxorubicin plus carboplatin (PLDC): a randomized phase III trial of the NOGGO-AGO-Study Group-AGO Austria and GEICOENGOT-GCIG intergroup study (HECTOR). Ann Oncol. 2016;27(12):2236-41.

\title{
Mammografie: Frauen mit dichter Brust bekommen nicht mehr Strahlung ab
}

\begin{abstract}
Frauen mit besonders dichtem Brustgewebe müssen nicht fürchten, dass sie bei der Mammografie eine höhere Strahlendosis abbekommen als andere, wie eine US-Studie nahelegt.
\end{abstract}

D as Risiko eines strahlungsbedingten Mammakarzinoms ist bei der heutigen Mammografietechnik relativ gering. Dennoch sind viele Frauen skeptisch, besonders wenn sie erfahren, dass sie „dichtes Brustgewebe“ haben, welches für sich genommen ein erhöhtes Brustkrebsrisiko birgt. Um herauszufinden, ob bei dichterem Brustgewebe auch mit einer höheren Strahlendosis zu rechnen ist, hat ein Team der University of Virginia Mammografiedaten von 434 Frauen (im Mittel 58 Jahre alt, BMI: 26,8) ausgewertet, die sich 2014 am radiologischen Zentrum einem Brustkrebsscreening unterzogen hatten. Dabei wurden zwei verschiedene digitale Mammografiegeräte eingesetzt, die Brustdichte und mittlere Parenchymdosis (AGD) messen konnten.

Weniger als ein Drittel der Frauen hatte dichtes oder sehr dichtes Brustgewebe. Die Dichtekategorien verteilten sich wie folgt: $28,8 \%$ hatten nahezu ausschließlich Fettgewebe (ACR 1), 42,6\% verstreute fibroglanduläre Verdichtungen (ACR 2), 23,5\% heterogen dichtes Gewebe mit einem Parenchymanteil zwischen 50 und $75 \%$ (ACR 3) und 5,1\% extrem dichtes Gewebe (ACR 4). Die volumenbezogene Brustdichte (VBD) betrug $5,8 \%$, die mittlere Dicke der komprimierten Brust („compressed breast thickness“, CBT) 63,4 mm. Je an der linken Brust wurde eine Parenchymdosis von 1,68 mGy erreicht, an der rechten Brust 1,75 mGy.

Die Variabilität der Strahlendosis war vor allem mit der komprimierten Brustdicke signifikant positiv assoziiert; dieser Parameter war für den Großteil (76\%) der erhaltenen Strahlungsdosis verantwortlich. Auf die Brustdichte entfielen nur $8 \%$. Ein höherer CBT-Wert resultiere in einer stärkeren Abschwächung der Röntgenstrahlung, so die For- scher. Das Gerät kompensiere dies automatisch, zum Beispiel durch eine höhere Röhrenspannung. Auch bei der „dichten Brust" komme es zu vermehrter Abschwächung im Drüsengewebe im Vergleich $\mathrm{zu}$ fettreichem Gewebe. Allerdings ergebe sich daraus - anders als bei großer CBT - nur ein geringer Effekt auf die Parenchymdosis. Bedenken in Bezug auf eine erhöhte Strahlungsdosis bei hoher Brustdichte sind für die Autoren daher unbegründet. Mit höherem Alter und mit höherem BMI nahm die Strahlendosis in der um die CBT bereinigten Analyse etwas ab; diese Zusammenhänge waren aber nicht signifikant.

Frauen mit hohem BMI neigten zwar zu dickeren Brüsten, aber auch zu einer geringeren Brustdichte. Diese Parameter beeinflussen die Strahlungsdosis individuell, so die Autoren. Sie schränken ein, dass die gerätespezifische Programmierung zudem zu Unterschieden in der Röhrenspannung, externen Filtration, Röhrenstromstärke und der Expositionsdauer führen könne.

Dr. Elke Oberhofer

Nguyen JV et al. Do women with dense breasts have higher radiation dose during screening mammography? Breast J 2017, online 7. Juni; doi: 10.1111/tbj.12833 